\title{
Effect of Enhanced External Counterpulsation and Cardiac Rehabilitation on Quality of Life, Plasma Nitric Oxide, Endothelin 1 and High Sensitive CRP in Patients With Coronary Artery Disease: A Pilot Study
}

\author{
Seyed Kazem Shakouri, MD¹, Zeynab Razavi, MD², Fariba Eslamian, MD, \\ Homayoun Sadeghi-Bazargani, $\mathrm{MD}^{3}$, Samad Ghaffari, $\mathrm{MD}^{2}$, Arash Babaei-Ghazani, $\mathrm{MD}^{4}$
}

${ }^{1}$ Physical Medicine and Rehabilitation Research Center, Tabriz University of Medical Sciences, Tabriz;

${ }^{2}$ Tabriz University of Medical Sciences, Tabriz; ${ }^{3}$ Injury Epidemiology \& Prevention Research Center, Statistics and Epidemiology Department, School of Health \& Nutrition, Tabriz University of Medical Sciences, Tabriz; ${ }^{4}$ Department of Physical Medicine and Rehabilitation, Iran University of Medical Sciences, Tehran, Iran

Objective To investigate the effect of enhanced external counterpulsation (EECP) on plasma nitric oxide (NO), Endothelin 1 (ET1), high sensitive C-reactive protein (HSCRP) and quality of life (QoL) in patients with coronary artery disease (CAD).

Methods We conducted a pilot randomized clinical trial in order to evaluate plasma NO, ET1, HSCRP and QoL before and after twenty sessions of EECP (group A) and cardiac rehabilitation (CR, group B) in 42 patients with CAD (21 in each group).

Results Forty-two patients ( 33 male and 9 female) were included in the study. The mean age was $58.2 \pm 10$ years. The mean HSCRP was $1.52 \pm 0.7$ in the EECP group and it was reduced to $1.27 \pm 0.4$ after intervention. The reduction in HSCRP was not statistically significant in EECP and CR groups with $\mathrm{p}=0.33$ and $\mathrm{p}=0.27$, respectively. There was not significant improvement of NO, ET1, and QoL in the EECP and CR groups shortly after therapy ( $>>0.05$ ).

Conclusion Although the short-term EECP treatment in CAD patients improved HSCRP, NO, ET1, and QoL compared with the baseline those improvements are not statistically significant. Further studies are necessary with large study groups and more sessions.

Keywords Enhanced external counter pulsation (EECP), Coronary artery disease, Nitric oxide, Endothelins, Highsensitivity C-reactive protein

(c) This is an open-access article distributed under the terms of the Creative Commons Attribution Non-Commercial License (http://creativecommons.org/ licenses/by-nc/3.0) which permits unrestricted noncommercial use, distribution, and reproduction in any medium, provided the original work is properly cited. Copyright $\odot 2015$ by Korean Academy of Rehabilitation Medicine 


\section{INTRODUCTION}

Coronary artery disease (CAD) is a leading cause of death and disability worldwide. An estimated 17.6 million patients in the United States suffer from symptomatic CAD and despite optimal medical therapy, such as medications, percutaneous coronary intervention, and cardiac bypass surgery, there are an estimated up to 900,000 patients who suffer from disabling angina $[1,2]$.

Despite these advances in surgical and interventional cardiology, up to a third of the patients with symptomatic CAD are not suitable for or cannot be fully revascularized [3].

Enhanced external counterpulsation (EECP) is a nonpharmacologic therapy for outpatients with angina pectoris and chronic heart failure (CHF). It is based on the principle of diastolic augmentation to increase the coronary flow while simultaneously decreasing the systolic afterload. During diastole, cuffs inflate sequentially from the calves proximally to raise the diastolic aortic pressure and theoretically increase the coronary perfusion pressure. At the same time, an increased venous return would result in an increased cardiac output by Frank-Starling mechanism. Then, the cuffs are rapidly decompressed at the onset of systole creating a negative pressure that decreases the cardiac afterload. EECP is a low risk procedure and approved by the US Food and Drug Administration for refractory angina and CHF [4].

Atherothrombosis is recognized as a dynamic chronic inflammatory process of the vessel wall in which phases of inflammatory and thrombotic activity underlie the clinical presentation of acute coronary syndromes (ACS). Inflammatory markers, such as high-sensitivity C-reactive protein (HSCRP) provide an alternative method for the global assessment of cardiovascular risk. Studies have shown that plasma levels of HSCRP are a strong independent risk predictor of future vascular events among individuals with and without known cardiovascular disease, including those patients who have been recently discharged from an ACS. Elevated levels of HSCRP have been positively correlated with most cardiovascular risk factors and have a strong association with adiposity and to a lesser extent to physical inactivity [5].

The endothelial dysfunction has been implicated as a major event in the pathogenesis of atherosclerosis. The plasma endothelin in CAD patients is higher whereas the nitric oxide (NO) level is significantly lower than in nor- mal people. Endothelin and NO may be used for the risk assessment especially in premature CAD and in individuals where traditional risk factors are not present [6].

Nevertheless, the impact of EECP treatment on HSCRP, Endothelin 1 (ET1), and NO in cardiac patients has not been well documented yet. The objective of this study was to investigative assess the role of EECP on these biomarkers of endothelial dysfunction and inflammation in CAD patients.

\section{MATERIALS AND METHODS}

\section{Study design and participants}

This pilot study was designed as a randomized controlled trial. Between April 2012 and March 2013, 42 consecutive patients with CAD were assessed for their suitability for inclusion in this study. Patients were referred to the physical medicine and rehabilitation clinic (cardiac rehabilitation center) of Madani Hospital. The assessor and the data analyzer were blinded, but the rehabilitation team conducting the interventions could not be blinded. The same assessor who was blinded to the group allocation made all of the measurements for each participant before the randomization and at the end of study.

Inclusion criteria were as follows: at least 30 years of age; angina pectoris of Canadian Cardiovascular Society (CCS) class I to IV despite the appropriate medical therapy; CAD diagnosis based on angiography or electrocardiogram or exercise test or cardiac perfusion scan; post-myocardial infarction (MI) with following angioplasty; and ejection fraction of at least $30 \%$. Exclusion criteria were as follows: having unstable angina or acute myocardial infarction or decompensated CHF in the preceding last month; blood pressure greater than 180/110 $\mathrm{mmHg}$; severe symptomatic peripheral vascular disease; pacemaker; having arrhythmias interfering with EECP function; active thrombophlebitis in lower limbs; bleeding diathesis and an international normalized ratio (INR) higher than 5; aortic aneurysm with surgery indication; pregnancy; severe valvular heart disease; history of nitroglycerin medications, such as nitrocantin in the last 2 weeks; history or symptoms of rheumatologic disease and any symptoms of infectious disease such as fever.

After a detailed explanation of the study protocol, a written informed consent was obtained from each eligible participant. The study was approved by the local research ethics committee and all procedures were con- 
ducted in accordance with the Declaration of Helsinki.

Patients were sequentially allocated into two parallel groups (A and B) with 21 participants in each group. Group A underwent EECP for 20 sessions (3 times per week). Each EECP session included one hour of EECP with Electrocardiogram monitoring. Group B had 20 sessions (3 times per week) of classic cardiac rehabilitation exercise protocol consisting of approximately 10 minutes of warm-up and light exercise (stretching) followed by 20 minutes of aerobic exercise including walking (treadmill) and bicycling and finally 5 to 10 minutes cool down period (stretching). The exercise intensity was individually prescribed so that the patient heart rates of each patient reached to approximately $60 \%$ to $80 \%$ (gradually increased) of the maximum heart rate calculated after exercise stress test. Before entering the program, patients underwent an exercise testing and we estimated the exercise capacity.

Socio-demographic information regarding age, sex, education status, employment, body mass index (BMI), familial history of CAD and risk factors, such as hypertension, chronic renal failure, and diabetes mellitus were collected at the baseline.

All subjects underwent an initial evaluation of main outcome measures including quality of life (QoL), CCS class, NO, HSCRP, and ET1 levels before and after intervention.

The QoL SF-36 is a widely used questionnaire for measuring a self-reported physical and mental health status. This questionnaire consists of 36 items measuring physical and mental health status in relation to eight health concepts: physical functioning, role limitations due to physical health, bodily pain, general health perceptions, vitality (energy/fatigue), social functioning, role limitations due to emotional health, and general mental health (psychological distress/wellbeing). Responses to each of the SF-36 items are expressed as a score on a 0-100 scale for each of the eight health concepts. Higher scores represent better self-perceived health [7].

The determination of NO, ET1, and HSCRP was performed with obtaining $5 \mathrm{~mL}$ of venous blood without adding any heparin. All blood samples were immediately centrifuged with 2,000 to 3,000 rounds per minute (rpm) at room temperature. The serum was extracted and restored at $-70^{\circ} \mathrm{C}$ until further use.

High-sensitivity assays for C-reactive protein were per- formed according to methods described by the manufacturer (Dade Behring Inc., Deerfield, IL, USA) [8].

The determination of NO in plasma was indirectly performed by the measurement of the stable decomposition product nitrite $\left(\mathrm{NO}_{2}\right)$, employing the Griess reaction [6]. Plasma endothelin was estimated by ELISA using DRG's human ET1 Enzyme Immunemetric Assay kit [6].

\section{Statistical analysis}

Statistical analysis was performed using the SPSS (ver. 16.0) statistical software (SPSS Inc., Chicago, IL, USA). Descriptive statistics were used, providing the numbers and percentages for the categorical variables and the means, standard deviations, minimum and maximum values for the numeric variables. Independent group comparisons were conducted using the Mann-Whitney U test when the data were assumed not to be normally distributed. The Friedman test was used for the repeatedmeasures analysis. The statistical significance was defined as a p-value of $<0.05[9]$.

\section{RESULTS}

Our study population was comprised of 42 patients with documented CAD, 21 patients in the EECP group and 21 in the CR exercise group. The EECP group was composed of 16 men and 5 women with a mean age of $61.8 \pm 9.5$ years, while the CR group was composed of 17 men and 4 women with a mean age of $64.6 \pm 9.4$ years. In each of EECP and CR groups, one patient at first week was lost to follow-up due to personal reasons.

The EECP group and the CR group did not differ in demographic characteristics at baseline $(p>0.05)$ (Table 1$)$.

A familial history of CAD in first-degree relatives was positive in 7 cases (35\%) of the EECP group and in 3 cases (15\%) of the CR group, but was statistically not significant $(\mathrm{p}=0.27)$. Four patients $(20 \%)$ in the EECP group and 8 (40\%) in the CR group had a history of smoking, but this was statistically not significant $(p=0.30)$. None of the patients were current smokers.

Clinical parameters including the QoL SF-36 questionnaire with eight subscales CCS class as well as laboratory findings of NO, HSCRP, and ET1 levels between two groups before and after intervention are shown in Tables 2 and 3. 
Table 1. Clinical characteristics of EECP and CR group

\begin{tabular}{lccc} 
& EECP & CR & p-value \\
\hline Hypertension & $8(38)$ & $8(38)$ & 1 \\
Diabetes & $4(19)$ & $7(33)$ & 0.48 \\
Hyperlipidemia & $8(38)$ & $7(33)$ & 1 \\
Weight $(\mathrm{kg})$ & $74.6 \pm 7.9(64-90)$ & $74.5 \pm 8.0(64-93)$ & 0.96 \\
Height $(\mathrm{cm})$ & $171.1 \pm 8.4(155-185)$ & $170.6 \pm 7.8(155-183)$ & 0.84 \\
Body mass index $\left(\mathrm{kg} / \mathrm{m}^{2}\right)$ & $25.6 \pm 2.6(21.6-31.5)$ & $24.5 \pm 2.7(20.4-32.6)$ & 0.98 \\
Left ventricle ejection fraction $(\%)$ & $45.2 \pm 10.8(30-65)$ & $44.2 \pm 9.3(30-60)$ & 0.75 \\
\hline
\end{tabular}

Values are presented as the number of patients per group (the percentage within each group) or mean \pm standard deviation (range). There were no significant differences $(p>0.05)$ in baseline characteristics between EECP and CR group. EECP, enhanced external counterpulsation; CR, cardiac rehabilitation.

Table 2. Comparison of the EECP and CR group in QoL and biochemical markers at baseline

\begin{tabular}{lccc}
\hline \multicolumn{1}{c}{ Variable } & $\begin{array}{c}\text { EECP } \\
(\mathbf{n = 2 0})\end{array}$ & $\begin{array}{c}\text { CR } \\
(\mathbf{n = 2 0})\end{array}$ & p-value \\
\hline CCS angina & & & 0.20 \\
I & $1(5)$ & $4(20)$ & \\
\hline II & $11(55)$ & $12(60)$ & \\
III & $8(40)$ & $4(20)$ & \\
IV & - & - & \\
QoL-SF36 & & & \\
$\quad$ Total score & $64.9 \pm 3.2$ & $66.0 \pm 3.7$ & 0.81 \\
\hline Subscales & & & \\
Physical function & $71.9 \pm 4.5$ & $73.0 \pm 20.4$ & 0.87 \\
Physical health & $58.5 \pm 8.3$ & $62.5 \pm 8.0$ & 0.73 \\
Emotional problem & $63.1 \pm 8.6$ & $64.9 \pm 9.8$ & 0.97 \\
Energy & $56.6 \pm 5.1$ & $61.0 \pm 4.1$ & 0.51 \\
Well being & $65.8 \pm 4.3$ & $65.4 \pm 3.0$ & 0.93 \\
Social function & $67.5 \pm 4.1$ & $76.8 \pm 4.1$ & 0.11 \\
Pain & $72.1 \pm 4.7$ & $65.0 \pm 30.5$ & 0.39 \\
General health & $63.9 \pm 3.5$ & $60.2 \pm 4.6$ & 0.76 \\
NO level & $7.87 \pm 0.99$ & $8.28 \pm 6.49$ & 0.81 \\
Endothelin 1 level & $7.49 \pm 2.81$ & $4.58 \pm 5.11$ & 0.34 \\
\hline HSCRP level & $1.52 \pm 0.7$ & $1.98 \pm 0.6$ & 0.62 \\
\hline Values are presented
\end{tabular}

Values are presented as number (\%) or mean \pm standard deviation.

EECP, enhanced external counterpulsation; CR, cardiac rehabilitation; QoL, quality of life; CCS, Canadian Cardiovascular Society; NO, nitric oxide; HSCRP, high sensitive C-reactive protein.

EECP and CR effect on clinical parameters and laboratory findings of CAD

Although there was an improvement in clinical and
Table 3. Comparison of the EECP and CR group in QoL and biochemical markers after the intervention

\begin{tabular}{lccc}
\hline \multicolumn{1}{c}{ Variable } & EECP $(\mathbf{n = 2 0})$ & $\mathbf{C R}(\mathbf{n = 2 0})$ & p-value \\
\hline CCS angina & & & 0.05 \\
I & $3(15)$ & $10(50)$ & \\
II & $16(80)$ & $9(45)$ & \\
III & $1(5)$ & $1(5)$ & \\
IV & - & - & \\
QoL-SF36 & & & \\
$\quad$ Total score & $65.4 \pm 3.5$ & $69.8 \pm 3.5$ & 0.38 \\
Subscales & - & - & \\
Physical function & $71.6 \pm 4.4$ & $76.7 \pm 4.5$ & 0.42 \\
Physical health & $58.9 \pm 7.9$ & $63.7 \pm 8.9$ & 0.66 \\
Emotional problem & $64.3 \pm 7.5$ & $75.5 \pm 34$ & 0.34 \\
Energy & $57.8 \pm 5.6$ & $61.7 \pm 4.2$ & 0.58 \\
Well being & $68.4 \pm 4.2$ & $65 \pm 3.6$ & 0.54 \\
Social function & $66.2 \pm 3.9$ & $77.7 \pm 4.4$ & 0.06 \\
Pain & $73.8 \pm 5$ & $71 \pm 6.4$ & 0.73 \\
General health & $61.9 \pm 3.3$ & $66.4 \pm 4.1$ & 0.65 \\
NO level & $8.21 \pm 1.27$ & $8.31 \pm 6.26$ & 0.78 \\
Endothelin 1 level & $5.22 \pm 0.6$ & $4.34 \pm 3.63$ & 0.88 \\
HSCRP level & $1.27 \pm 0.4$ & $1.75 \pm 0.6$ & 0.36 \\
\hline
\end{tabular}

Values are presented as number (\%) or mean \pm standard deviation.

EECP, enhanced external counterpulsation; CR, cardiac rehabilitation; QoL, quality of life; CCS, Canadian Cardiovascular Society; NO, nitric oxide; HSCRP, high sensitive C-reactive protein.

laboratory parameters in the pre-trial and post-trial comparison within both groups, there was not any statistically significant improvement over time (Tables 4,5 ).

Comparing the amount of improvement in total QoL 
Table 4. Effects of EECP on QoL and biochemical markers

\begin{tabular}{lccc}
\hline \multicolumn{1}{c}{ Variable } & $\begin{array}{c}\text { Pre-trial } \\
(\mathbf{n}=20)\end{array}$ & $\begin{array}{c}\text { Post-trial } \\
(\mathbf{n = 2 0})\end{array}$ & p-value \\
\hline QoL-SF36 & & & \\
$\quad$ Total score & $64.9 \pm 3.2$ & $65.4 \pm 3.5$ & 0.54 \\
$\quad$ Subscales & - & - & \\
Physical function & $71.9 \pm 4.5$ & $71.6 \pm 4.4$ & 0.83 \\
Physical health & $58.5 \pm 8.3$ & $58.9 \pm 7.9$ & 1.00 \\
Emotional problem & $63.1 \pm 8.6$ & $64.3 \pm 7.5$ & 1.00 \\
Energy & $56.6 \pm 5.1$ & $57.8 \pm 5.6$ & 0.66 \\
Well being & $65.8 \pm 4.3$ & $68.4 \pm 4.2$ & 0.09 \\
Social function & $67.5 \pm 4.1$ & $66.2 \pm 3.9$ & 0.69 \\
Pain & $72.1 \pm 4.7$ & $73.8 \pm 5.0$ & 0.54 \\
General health & $63.9 \pm 3.5$ & $61.9 \pm 3.3$ & 0.38 \\
NO level & $7.87 \pm 0.99$ & $8.21 \pm 1.27$ & 0.63 \\
Endothelin 1 level & $7.49 \pm 2.81$ & $5.22 \pm 0.60$ & 0.43 \\
HSCRP level & $1.52 \pm 0.7$ & $1.27 \pm 0.4$ & 0.33 \\
\hline
\end{tabular}

Values are presented as mean \pm standard deviation.

EECP, enhanced external counterpulsation; QoL, quality of life; NO, nitric oxide; HSCRP, high sensitive C-reactive protein.

Table 5. Effects of CR on QoL and biochemical markers

\begin{tabular}{lccc}
\hline \multicolumn{1}{c}{ Variable } & $\begin{array}{c}\text { Pre-trial } \\
(\mathbf{n = 2 0})\end{array}$ & $\begin{array}{c}\text { Post-trial } \\
(\mathbf{n = 2 0})\end{array}$ & p-value \\
\hline QoL-SF36 & & & \\
$\quad$ Total score & $66.0 \pm 3.7$ & $69.8 \pm 3.5$ & 0.23 \\
\hline Subscales & & & \\
Physical function & $73.0 \pm 20.4$ & $76.7 \pm 4.5$ & 0.17 \\
Physical health & $62.5 \pm 8.0$ & $63.7 \pm 8.9$ & 0.89 \\
Emotional problem & $64.9 \pm 9.8$ & $75.5 \pm 34.0$ & 0.39 \\
Energy & $61.0 \pm 4.1$ & $61.7 \pm 4.2$ & 0.81 \\
Well being & $65.4 \pm 3.0$ & $65.0 \pm 3.6$ & 0.90 \\
Social function & $76.8 \pm 4.1$ & $77.7 \pm 4.4$ & 0.85 \\
Pain & $65 \pm 30.5$ & $71 \pm 6.4$ & 0.28 \\
General health & $60.2 \pm 4.6$ & $66.4 \pm 4.1$ & 0.09 \\
NO level & $8.28 \pm 6.49$ & $8.31 \pm 6.26$ & 0.87 \\
Endothelin 1 level & $4.58 \pm 5.11$ & $4.34 \pm 3.63$ & 0.66 \\
HSCRP level & $1.98 \pm 0.6$ & $1.75 \pm 0.6$ & 0.27 \\
\hline
\end{tabular}

Values are presented as mean \pm standard deviation.

CR, cardiac rehabilitation; QoL, quality of life; NO, nitric oxide; HSCRP, high sensitive C-reactive protein.
Table 6. Comparison of EECP and CR group in the improvement of QoL and biochemical markers

\begin{tabular}{lccc}
\hline \multicolumn{1}{c}{ Variable } & $\begin{array}{c}\text { EECP } \\
\text { difference } \\
(\mathbf{n = 2 0})\end{array}$ & $\begin{array}{c}\text { CR } \\
\text { difference } \\
(\mathbf{n = 2 0})\end{array}$ & p-value \\
\hline $\begin{array}{l}\text { QoL-SF36 } \\
\text { Total score }\end{array}$ & $0.5 \pm 3.9$ & $3.8 \pm 3.6$ & 0.31 \\
\hline Subscales & - & - & \\
Physical function & $-0.3 \pm 1.3$ & $3.7 \pm 1.9$ & 0.18 \\
Physical health & $0.4 \pm 4.2$ & $1.2 \pm 4.7$ & 0.90 \\
Emotional problem & $1.2 \pm 1.1$ & $10.6 \pm 14.8$ & 0.40 \\
Energy & $1.2 \pm 1.6$ & $0.7 \pm 14.3$ & 0.92 \\
Well being & $2.6 \pm 6.4$ & $-0.4 \pm 4.9$ & 0.42 \\
Social function & $-1.3 \pm 3.9$ & $0.9 \pm 2.6$ & 0.70 \\
Pain & $1.7 \pm 1.6$ & $6.2 \pm 2.4$ & 0.47 \\
General health & $-2.0 \pm 9.9$ & $6.2 \pm 15.9$ & 0.32 \\
NO level & $0.34 \pm 0.03$ & $0.03 \pm 0.82$ & 0.67 \\
Endothelin 1 level & $2.27 \pm 12.6$ & $0.24 \pm 2.3$ & 0.48 \\
HSCRP level & $0.25 \pm 1.1$ & $0.23 \pm 0.9$ & 0.93 \\
\hline Values are presented & & & \\
\hline
\end{tabular}

Values are presented as mean \pm standard deviation.

EECP, enhanced external counterpulsation; CR, cardiac rehabilitation; QoL, quality of life; NO, nitric oxide; HSCRP, high sensitive C-reactive protein.

SF-36 and its eight subscales, NO level, ET1 level, and HSCRP level, there was no statistical significance between both groups (Table 6).

\section{DISCUSSION}

This randomized clinical trial-pilot study demonstrates for the first time effects of EECP on inflammatory parameters of NO, ET1, and HSCRP and QoL in patients with CAD. We hypothesized that 20 sessions EECP treatment could improve inflammatory parameters of CAD patients.

The present study showed that 20 sessions of EECP did not significantly improve the QoL, NO, ET1, and HSCRP level.

As an effective noninvasive treatment method for patients with CAD, EECP has been proven to exert various beneficial effects on the cardiovascular system, such as increasing the coronary perfusion pressure and arterial wall shear stress $[10,11]$.

Benefits associated with EECP include the reduction of angina and nitrate use, an increased exercise tolerance, favorable psychosocial effects and an enhanced QoL as 
well as a prolongation of the time to exercise-induced STsegment depression and an accompanying resolution of myocardial perfusion defects [12].

The mechanisms of EECP responsible for improving the coronary blood flow are unclear. Numerous studies have identified several possible mechanisms of action of EECP. Initially, the prevailing theory was that EECP increases the coronary collateral circulation [13].

The safety of EECP treatment was demonstrated with minor adverse effects, such as skin blebs in patients with left ventricular dysfunction [4].

In different conditions and diseases showed EECP promising results. A prospective evaluation on the EECP in congestive heart failure showed an improved exercise tolerance and QoL due to EECP treatment [14]. Liu et al. [15] demonstrated that, even though the mean blood pressure did not significantly change, EECP treatment is associated with a significant elevation in the right common carotid artery blood flow. Lin et al. [16] reported that EECP was able to improve the mean blood pressure and cerebral perfusion in patients after ischemic stroke. Another study concluded that angina patients with aortic stenosis who underwent EECP had clinically important symptomatic and hemodynamic improvements comparable to their non-aortic stenosis counterparts [17]. Ruangkanchanasetr et al. [4] even demonstrated that EECP could improve the long-term renal function in cardiac patients especially in cases with declined renal function.

Previous studies have shown that elevated inflammatory biomarkers are predictive of future peripheral arterial diseases, $\mathrm{CAD}$ and cerebrovascular accidents and any improvement in these markers could halt CAD progression $[6,18,19]$. HSCRP increased in baseline samples of subjects who subsequently developed peripheral arterial disease compared with controls and the calculated relative risks for CAD increased significantly with each increasing quartile of measured HSCRP [8].

Therapeutic lifestyle changes affected through a threemonth cardiac rehabilitation program significantly improved numerous cardiac risk factors. With this approach to a secondary prevention, Milani et al. [5] observed significant reductions in HSCRP levels. These findings identify another clinical modality of reducing HSCRP beyond use of statin drugs and suggest an additional benefit of formal phase II cardiac rehabilitation and exercise training programs.
Comparing in our study, the absence of a significant improvement shows that 20 sessions EECP is not effective. However, we would not come to the conclusion to rule out EECP effects on CAD patients. Our study shows a non-significant improvement of inflammatory biomarkers from baseline values and this could be an indication of possible significant results in case of continuing EECP beyond twenty sessions.

Also, in the conventional CR group we did not find a significant improvement of inflammatory biomarkers. Based on the pilot nature of our study, these finding could be explained with different hypothesizes; first, we would hypothesize that inflammatory biomarkers are not sensitive outcome measures for the cardiac rehabilitation assessment and that they could not rule in or rule out any cardiac rehabilitation method. Second, our study is a pilot study and our sample size could be too small. Third, there is not definitive and worldwide accepted protocol for CR sessions and these results could simply indicate that this protocol has no effect on inflammatory biomarkers of CAD patients. We need focused studies on these subjects for a definite conclusion on this matter.

Heffernan et al. [20] investigated the effect of resistance training on systemic inflammatory biomarkers such as CRP in African-Americans compared with White men. They found different responses between these two ethnic groups which could suggest the importance of ethnicity in the response of inflammatory biomarkers to exercise training.

Whether racial differences are truly biological or merely reflect differences in environmental factors (i.e., diet, social support) and/or psychosocial factors (i.e., perceived stress, discrimination, coping ability, and locus of control) is beyond the scope of this study.

We did not include psychological and dietary management in our interventions and this could be another influencing factor. Also subject's stress levels were not controlled during this study what may have influenced overall findings.

There are different factors effecting conventional CR results like choice of outcome measures for improvement evaluation, age, sex, BMI, time of biochemical blood sampling after CR, sample size and ethnicity of subject group.

Taking all trials together, it may be speculated that a training period of only 7 weeks, as preferred in our study, 
may not be long enough to induce changes in inflammatory biomarkers, since most studies were carried out for 12 to 26 weeks. These results do not exclude beneficial effects of conventional CR or EECP in CAD patients, but it undermines effects of 7-week conventional CR and EECP program on biochemical markers in CAD patients.

It should be highlighted that our non-significant improvement results in both groups are not implying an ineffectiveness of EECP or conventional CR in 20 sessions. This study only shows that we should not expect significant change in biochemical markers with 20 sessions of EECP or conventional CR. Also we should mention that our results imply to the ethnicity of Azeri people with their regional diet and living in a higher altitude from sea level.

Our pilot study included twenty sessions of EECP and CR. The main limitations of our study are the small numbers of rehabilitation sessions and the small sample size. Based on mild but not significant improvements, more sessions might show significant results. So, further studies should be conducted with more sessions of EECP and larger groups of study subjects.

In conclusion, twenty sessions of EECP treatment had no significant effect on the improvement of QoL, NO, ET1, and HSCRP in CAD patients. Further studies are necessary with more sessions of EECP and a larger sample size with controls.

\section{CONFLICT OF INTEREST}

No potential conflict of interest relevant to this article was reported.

\section{REFERENCES}

1. Soran O, Ikizler C, Sengul A, Cuglan B, Kennard E, Kelsey S. Comparison of long term clinical outcomes, event free survival rates of patients undergoing enhanced external counterpulsation for coronary artery disease in the United States and Turkey. Turk Kardiyol Dern Ars 2012;40:323-30.

2. Kim C, Youn JE, Choi HE. The effect of a self exercise program in cardiac rehabilitation for patients with coronary artery disease. Ann Rehabil Med 2011;35:381-7.

3. Loh PH, Kennard E, Bourantas CV, Chelliah R, Atkin
P, Cook J, et al. The effectiveness of enhanced external counterpulsation (EECP) in patients suffering from chronic refractory angina previously treated with transmyocardial laser revascularisation. Int J Cardiol 2013;168:4383-5.

4. Ruangkanchanasetr P, Mahanonda N, Raungratanaamporn O, Ruckpanich P, Kitiyakara C, Chaiprasert A, et al. Effect of enhanced external counterpulsation treatment on renal function in cardiac patients. BMC Nephrol 2013;14:193.

5. Milani RV, Lavie CJ, Mehra MR. Reduction in C-reactive protein through cardiac rehabilitation and exercise training. J Am Coll Cardiol 2004;43:1056-61.

6. Saini V, Bhatnagar MK, Bhattacharjee J. Association of endothelial dysfunction with endothelin, nitric oxide and eNOS Glu298Asp gene polymorphism in coronary artery disease. Dis Markers 2011;31:215-22.

7. Montazeri A, Goshtasebi A, Vahdaninia M, Gandek B. The Short Form Health Survey (SF-36): translation and validation study of the Iranian version. Qual Life Res 2005;14:875-82.

8. Rifai N, Tracy RP, Ridker PM. Clinical efficacy of an automated high-sensitivity C-reactive protein assay. Clin Chem 1999;45:2136-41.

9. Eftekhar Sadat B, Khadem Haghighian M, Alipoor B, Malek Mahdavi A, Asghari Jafarabadi M, Moghaddam A. Effects of sesame seed supplementation on clinical signs and symptoms in patients with knee osteoarthritis. Int J Rheum Dis 2013;16:578-82.

10. Braith RW, Conti CR, Nichols WW, Choi CY, Khuddus MA, Beck DT, et al. Enhanced external counterpulsation improves peripheral artery flow-mediated dilation in patients with chronic angina: a randomized sham-controlled study. Circulation 2010;122:1612-20.

11. Bonetti PO, Barsness GW, Keelan PC, Schnell TI, Pumper GM, Kuvin JT, et al. Enhanced external counterpulsation improves endothelial function in patients with symptomatic coronary artery disease. J Am Coll Cardiol 2003;41:1761-8.

12. Bonetti PO, Holmes DR Jr, Lerman A, Barsness GW. Enhanced external counterpulsation for ischemic heart disease: what's behind the curtain? J Am Coll Cardiol 2003;41:1918-25.

13. Braverman DL. Enhanced external counterpulsation: a novel therapy for angina. Complement Ther Clin Pract 2012;18:197-203. 
14. Feldman AM, Silver MA, Francis GS, Abbottsmith CW, Fleishman BL, Soran O, et al. Enhanced external counterpulsation improves exercise tolerance in patients with chronic heart failure. J Am Coll Cardiol 2006;48:1198-205.

15. Liu R, Liang ZJ, Liao XX, Hu CL, Jiang L, Dai G, et al. Enhanced external counterpulsation improves cerebral blood flow following cardiopulmonary resuscitation. Am J Emerg Med 2013;31:1638-45.

16. Lin W, Xiong L, Han J, Leung TW, Soo YO, Chen X, et al. External counterpulsation augments blood pressure and cerebral flow velocities in ischemic stroke patients with cerebral intracranial large artery occlusive disease. Stroke 2012;43:3007-11.

17. Braverman DL, Braitman L, Figueredo VM. The safety and efficacy of enhanced external counterpulsation as a treatment for angina in patients with aortic stenosis. Clin Cardiol 2013;36:82-7.

18. Ferreiros ER, Boissonnet CP, Pizarro R, Merletti PF, Corrado G, Cagide A, et al. Independent prognostic value of elevated C-reactive protein in unstable angina. Circulation 1999;100:1958-63.

19. Cooke JP. Role of nitric oxide in progression and regression of atherosclerosis. West J Med 1996;164:41924.

20. Heffernan KS, Jae SY, Vieira VJ, Iwamoto GA, Wilund $\mathrm{KR}$, Woods JA, et al. C-reactive protein and cardiac vagal activity following resistance exercise training in young African-American and white men. Am J Physiol Regul Integr Comp Physiol 2009;296:R1098-105. 